\title{
The use of multiple independent gains for a repetitive low frequency duty cycle in a hydraulic system
}

\author{
R.T BURTON, C.M. SARGENT, G.J. SCHOENAU \\ AND D. ANDERSON \\ University of Saskatchewan \\ Saskatoon, SK \\ Canada S7N OW0
}

\begin{abstract}
To produce a desired wave form, the required input control signal to a system must be known. If, for a repetitive duty cycle, the output and input signals of the system may be represented by quantized patterns, then the relationship between the input and output at any given point of the pattern can be considered as a simple gain. Thus, multiple independent gains (MIG) can be used to define the complete pattern. If the gains at each point can be determined, the output of the system can be made to follow the desired pattern regardless of the linear or nonlinear nature of the system. This concept has been applied to direct both a linear analog system as well as an electrohydraulic system to follow a specified low frequency $(0.1 \mathrm{~Hz})$ repetitive pattern. The paper concludes with a discussion of the limitations and potential of the MIG concept.
\end{abstract}

\section{KEYWORDS}

Hydraulic servo system, multiple gains, pattern reproduction

\section{NOMENCLATURE}

i subscript indicating $i$ th location in the pattern

$\mathrm{k} \quad$ index for $\mathrm{k}$ th training cycle

$e_{i}(k) \quad$ error between desired and actual output

$\mathrm{J}_{\mathrm{i}}(\mathrm{k}) \quad$ cost function

$\mathrm{K}_{\mathrm{i}}(\mathrm{k})$ gain at the $\mathrm{i}$ th location

$\mathrm{U}_{\mathrm{i}}(\mathrm{k}) \quad$ input to plant for $\mathrm{k}$ th training cycle

$X_{\text {di }} \quad$ desired plant output

$\mathrm{X}_{\mathrm{Oi}}(\mathrm{k})$ actual plant output for $\mathrm{k}$ th cycle

$\mu \quad$ gain adjustment constant

$\beta_{\mathrm{i}}(\mathrm{k}) \quad$ slope for Newton's method

$\alpha \quad$ gradient factor

\section{INTRODUCTION}

Frequently, the task that a hydraulic system has to perform is repetitive and of relatively low frequency so that the speed of response of the hydraulic system is of secondary importance, and the main problem is the nonlinearity of the system (1). Traditional control strategies are oriented towards improving the speed of response, desensitizing the output of the system to noise disturbances and adapting to variations in system parameters. All control strategies have encountered difficulties when severe non-linearities are present. Primarily this is because these control schemes are responsive in nature i.e. they must first detect the presence of the non-linearity and then respond to it. For a repetitive task this must be done every cycle.

In order to achieve a desired (output) waveform, the input to the hydraulic system must be related to the output by the inverse of the 
system. However, this requires that the system inverse be known. Determining the inverse of a non-linear system is not a trivial matter. Techniques which attempt to do this include system identification (2), model referencing (3) and, more recently neural networks (4).

Figure 1 shows schematically an example of a required input waveform that will produce a desired output pattern. At any non-zero point (e.g. A) in the cycle, the output can be regarded as being related to the input by a simple multiplying factor, i.e. a gain. If the required output waveform is quantized, then the problem of determining the system inverse can be circumvented by determining the appropriate multiplier for each point of the required output waveform. If this can be done for each point in the pattern then each gain can be regarded as independent. Thus the technique is referred to as the multiple independent gain (MIG) technique.

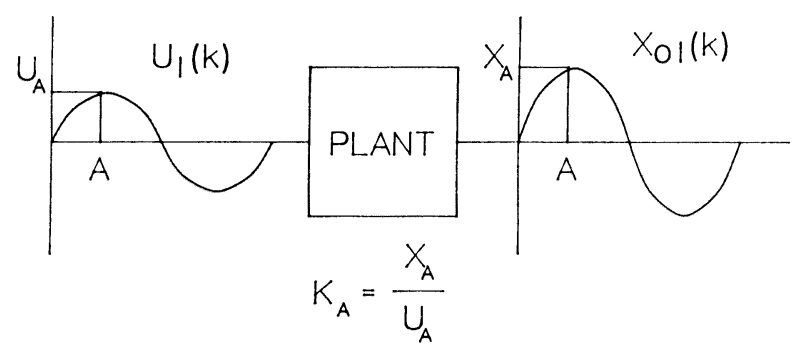

Figure 1. Input pattern required to produce the desired output pattern of a plant.

If the gains at each point can be determined, the output of the system can be made to approximate the desired pattern regardless of the nature of the system, that is the system can be linear or nonlinear. No prior knowledge of the system characteristics (static or dynamic) is required if the gains can be determined by minimizing techniques (such as least squared error) since these gain estimation techniques are error driven at each point in the pattern.

In practice, the "input" to the system (see Figure 1) represents a voltage to be sent to a servo valve and the "output" a voltage from a suitable transducer. The system is an open loop configuration. The gain at a point such as A, therefore, would include the effective gains of the servo valve and of the transducer at that point in the cycle. For a linear servo valve, transducer, and system, the gain for each point of the cycle would be constant. For a non-linear system, the gain is dependent not only on amplitude and frequency of the required pattern but also on the relative location of the nonlinearity within the pattern. If the characteristics of the system are readily available and invariant in time, it may be possible to estimate the appropriate gains $a b$ initio and store these in a computer to be fed sequentially at required intervals to the system.

It is the intention of this paper to compare the application of the MIG technique to a well defined analog system and to a "real" system with noise and nonlinearities such as those found in an inertially loaded electrohydraulic servo system.

\section{IMPLEMENTATION}

Figure 2 schematically represents the MIG technique. The desired input pattern is quantized $\left(\mathrm{X}_{\mathrm{di}}\right)$ and is input to the MIG driver. After appropriate adjustments of the gains, $\mathrm{K}_{\mathrm{i}}(\mathrm{k})$, the $\mathrm{MIG}$ output $\mathrm{U}_{\mathrm{i}}(\mathrm{k})$ is sequenced in time to produce a full cycle input to the plant. The output from the plant over one full cycle is converted back to a quantized pattern $\left(\mathrm{X}_{\mathrm{oi}}(\mathrm{k})\right)$ compatible with the desired input, $X_{\mathrm{di}}$. The two patterns $X_{\mathrm{oi}}(\mathrm{k})$ and $\mathrm{X}_{\mathrm{di}}$ are compared and the error over the pattern $e_{i}(k)$ is fed into the gain adjusting algorithm which uses the $e_{i}(k)$ error to adjust the $\mathrm{K}_{\mathrm{i}}(\mathrm{k})$ gain in the MIG driver. The adjustments of these gains will be discussed presently.

The experimental system

A hydraulic motor was loaded with a mass of $20 \mathrm{~kg}$ with a mass moment of inertia of $16 \mathrm{kgm}^{2}$. Flow to the motor was modulated using a Moog electrohydraulic servo valve. The velocity of the motor was monitored by a Schaevitz tachometer The output of the tachometer and the input to the servo valve were interfaced to a Amdex microcomputer via 12 bit $\mathrm{A} / \mathrm{D}$ and D/A converters (Dash 8) respectively. For all studies a quantized sinusoidal variation in velocity was the desired output of the plant. An open loop response to a sinusoidal input shows the degree of non-linearity that exists at zero velocity in the waveform (Figure 3).

The simulated system

To provide a means of comparison, a second order system with 0.77 damping ratio and a natural frequency of $22.8 \mathrm{rad} / \mathrm{sec}$ was simulated using an analog computer (Comdyna GP-6). These parameter values were chosen to approximately match the physical system. The analog model was interfaced to the same microcomputer used for the hydraulic system via the same 12 bit $A / D$ and D/A converters. For comparative purposes a quantized sinusoidal pattern was the desired output. 


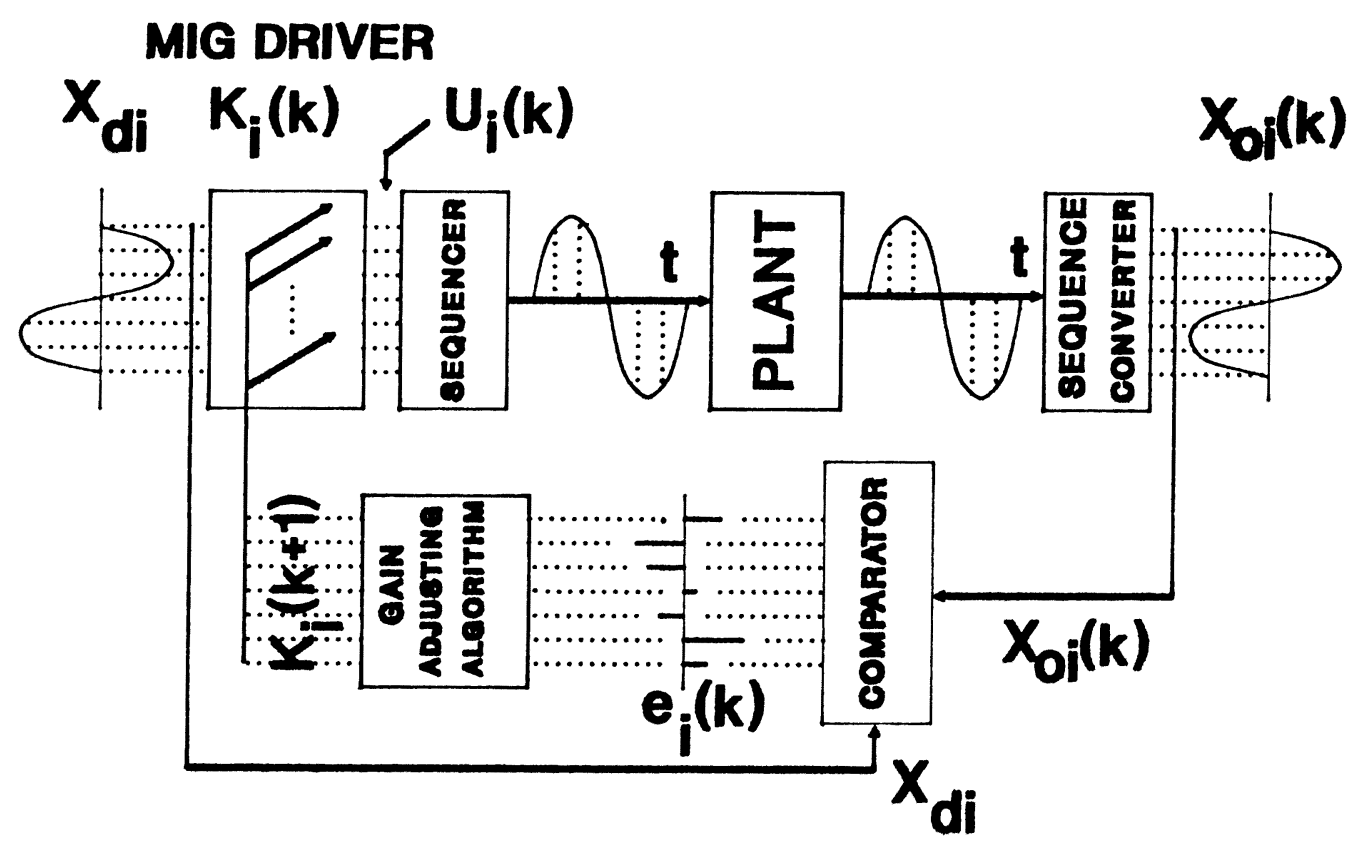

Figure 2 Overall system illustrating the MIG concept

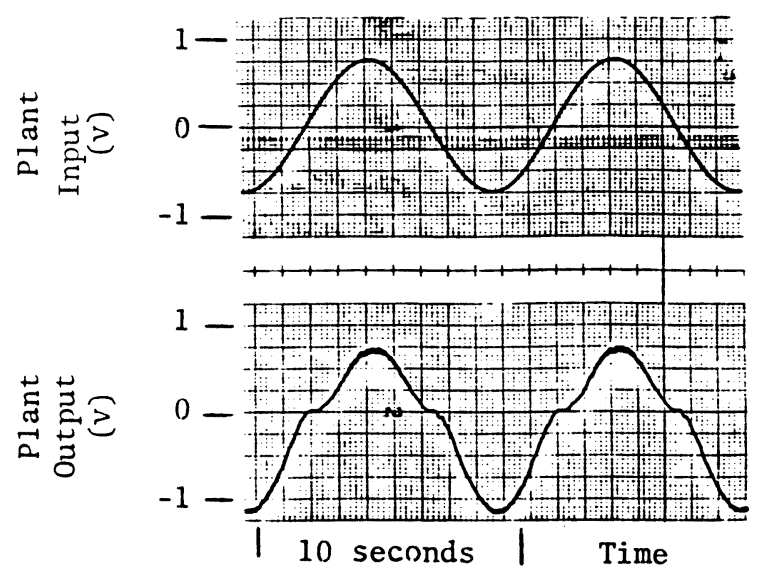

Figure 3. Non-linear open-loop characteristics of the plant for a sinusoidal input

\section{Adjustment of the gains}

For a linear system the gains can be computed from the characteristics of the system if known. If, however, the system characteristics are unknown, then some technique must be found to determine the appropriate gains. Two such techniques were used in this work - methods based on a least squared error approach and on Newton's method for determining the roots of an equation.

To illustrate the least squares approach, consider Figure 2. If $\mathrm{X}_{\mathrm{oi}}(\mathrm{k})$ is the output of the system and $X_{\mathrm{di}}$, the desired pattern at a training period $(\mathrm{k})$, then the error at the $\mathrm{i}$ th location in the pattern is simply:

$e_{i}(k)=X_{d i}-X_{o i}(k)$

The least mean squares approach is often encountered in minimizing problems. For this application, the cost function, $\mathrm{J}$, defined by the expectation of the square of the error, is minimized with respect to the feed-forward gains, $\mathrm{K}_{\mathrm{i}}(\mathrm{k})$.

$J_{i}(k)=\frac{1}{2} E\left(e_{i}(k)^{2}\right)$

Consequently, the change in the gains at the next training period is given by: 
$K_{i}(k+1)=K_{i}(k)-\mu \frac{\partial J_{i}(k)}{\partial K_{i}(k)}$

where

$\frac{\partial J_{i}(k)}{\partial K_{i}(k)}=-e_{i}(k) x_{d i}$

$X_{d i}$ is the input to the driver (See Figure 2) and $\mu$ is a gain adjustment constant.

To speed convergence, a momentum term is often added to yield:

$$
\begin{aligned}
K_{i}(k+1)= & K_{i}(k)-\mu \frac{\partial J_{i}(k)}{\partial K_{i}(k)} \\
& +\alpha\left(K_{i}(k)-K_{j}(k-1)\right)
\end{aligned}
$$

The second method to adjust the gains uses a modified versions of Newton's root solving technique to determine the correct input to the plant $\left(\mathrm{U}_{\mathrm{i}}\right)$ which will produce the desired output, $X_{\text {di }}$.The functional relationship between the plant input and output can be expressed as $X_{\mathrm{Oi}}=\mathrm{FU}_{\mathrm{i}}$, where $\mathrm{F}$ is an operator (gain) of the plant. This operator is unknown and graphically can be represented by a curve such as that illustrated in Figure 4. If two initial values $\left(U_{i}(1)\right.$ and $\left.U_{i}(2)\right)$ are input to the plant, then the slope, $\beta_{i}(2)$, of a straight line between the two points on the $X_{o i}-U_{i}(k)$ curve is given by:

$$
\beta_{i}(2)=\frac{X_{o i}(1)-X_{o i}(2)}{U_{i}(1)-U_{i}(2)}
$$

Thus a new value of $\mathrm{U}_{\mathrm{i}}$ is thus:

$$
U_{i}(3)=U_{i}(2)+\frac{X_{o i}(2)-X_{d i}}{\beta_{i}(2)}
$$

or in general

$$
U_{i}(k+1)=U_{i}(k)+\frac{X_{o i}(k)-X_{d i}}{\beta_{i}(k)}
$$

From $U_{i}$, a new $X_{o i}$, is found and the iteration process continues until $\mathrm{X}_{\mathrm{di}}-\mathrm{X}_{\mathrm{Oi}(\mathrm{k})}$ falls within an acceptable error band.

Presentation of results

The presentation of the results will be separated into two regimes: the first at a very low frequency $(0.1 \mathrm{~Hz})$ at which phase shifts are small; and the second at a higher frequency $(0.5$ $\mathrm{Hz}$ ) where a phase shift can become significant.

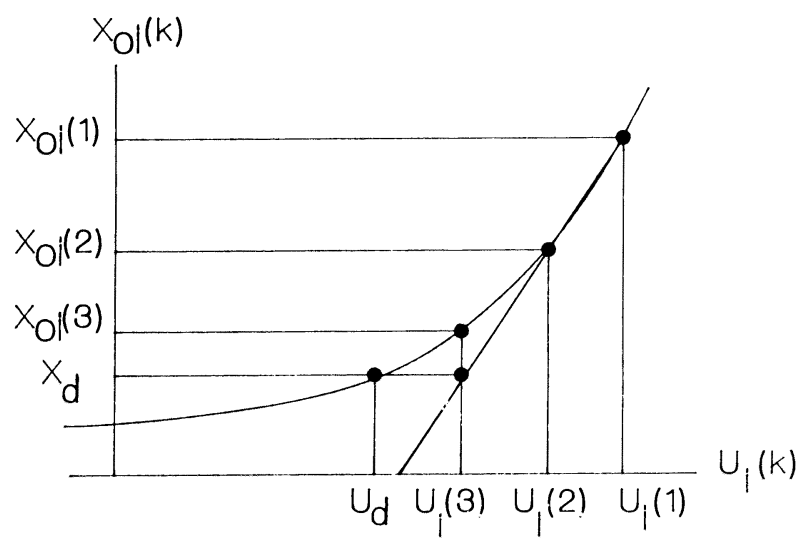

Figure 4 Modified Newton's method

Low frequency $(0.1 \mathrm{HZ})$

The results of both the least squares and the Newton's method for the simulated second order plant are shown in Figures 5(a) and (b) respectively. The output patterns converge rapidly to the desired pattern (about 15 iterations).

The results of both methods for the "real" system are shown in Figures 5(c) and (d) for the least squares and modified Newton method respectively. The least squares technique successfully converged to a stable pattern after a few iterations (Figure 5 (c)). The modified Newton's method converged to a stable solution, but was sensitive to noise. The non-linearities caused severe spiking during training and this technique was not used in the higher frequency tests. It must be noted that in both cases the non-linear dead zone at zero velocity as seen in Figure 3 has been almost completely overcome.

\section{Higher frequency $(0.5 \mathrm{HZ})$}

Figure 6 (a) and (b) of the results from the hydraulic and simulated analog systems show that a stable solution was not possible. Similar results were obtained using the modified Newton's method. At this frequency a phase shift of approximately $45^{\circ}$ was inherent to both systems. Although it is possible to find a set of gains which could reflect an exact solution to the problem of the phase shift, a method of 
converging to those gains cannot be found using the MIG technique. The reason is that the gains are independent and that for a phase shift, the effects of a change in gain are felt at a later time in the pattern. Some cross-coupling of gains is required to handle the phase shift problem.

\section{DISCUSSION}

An electro-hydraulic system was chosen for this study because it displays some very unique and severe non-linearities which are very difficult to compensate for when using traditional control strategies. The servo-valve and the inertially loaded actuator in their uncontrolled state displayed a nonlinear response when subjected to a sinusoidal input.

Both the simulated and hydraulic systems could learn to follow a specified low frequency $(0.1 \mathrm{~Hz})$ repetitive pattern using the least squares worked very well for the analog simulation in which the signal was "clean" and "wellbehaved". In the presence of noise and/or nonlinearities the technique sometimes failed to converge to the desired pattern. This shows the importance of using realistic models when evaluating a technique.

The problem of phase remains and for higher frequency operations where the phase shift is significant, the use of an artificial neural net which would allow "looking ahead" shows more promise. (4)

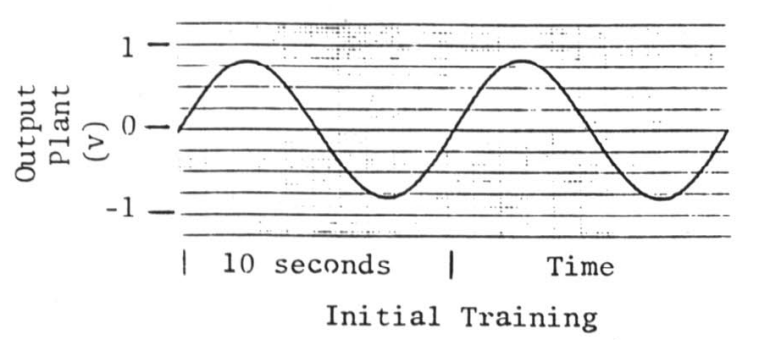
approach. The modified Newton's method

\section{CONCLUSION}

The multiple independent gain technique is a viable technique for directing a non-linear plant such as a hydraulic servo system to follow a low frequency repetitive duty cycle where the phase shift is not significant. No knowledge of the characteristics of the system is required and the convergence is rapid. The importance of this technique lies in its ability to significantly reduce the effects of non-linearities in the system.

\section{REFERENCES}

(1) Jannerup O.,"Integrated Analysis, Design and Simulation on Hydraulic Servo Systems", Proceedings of the 2nd Tampere International Conference on Fluid Power, Tampere, Finland, 1991.

(2) Widrow, B., and Winter, R. "Neural Nets for Adaptive Filtering and Adaptive Pattern Recognition", Computer Magazine, March, 1988.

(3) Hori, N., Ukrainetz, P.R., Nikiforuk, P.N. and Bitner, D.V., "Robust Discrete-Time Adaptive Control of an Electrohydraulic Servo Actuator", Fluid Power, Elsevier Applied Science Publishers, London, 1989.

(4) Burton, R.T., Sargent, C.M., and Schoenau, G.J., "Using an Artificial Neural Network to Direct a Hydraulic Circuit", Proc. of 43rd Inf. Conf. on Fluid Power (NCFP), Chicago, USA, 1992.

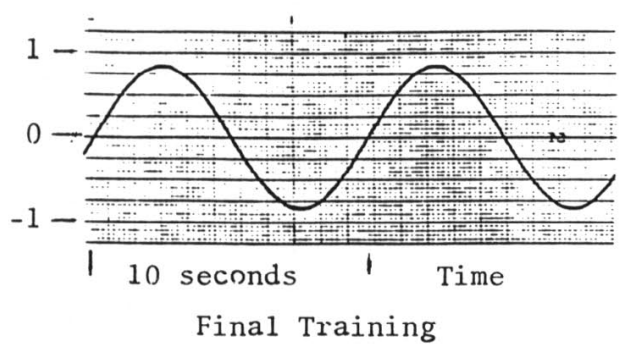

(a) Least Squared
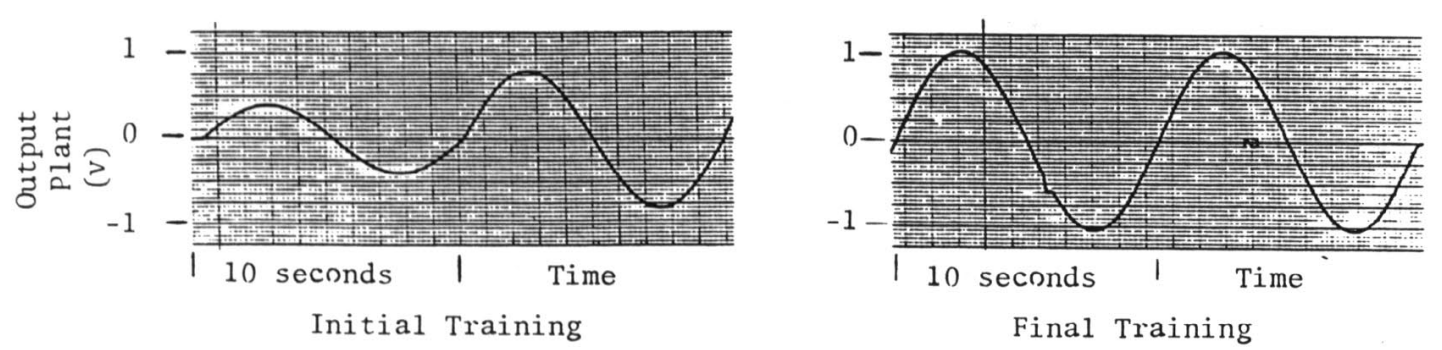

(b) Modified Newtons

Figure 5(a) and (b) Simulation Results (.1H) 


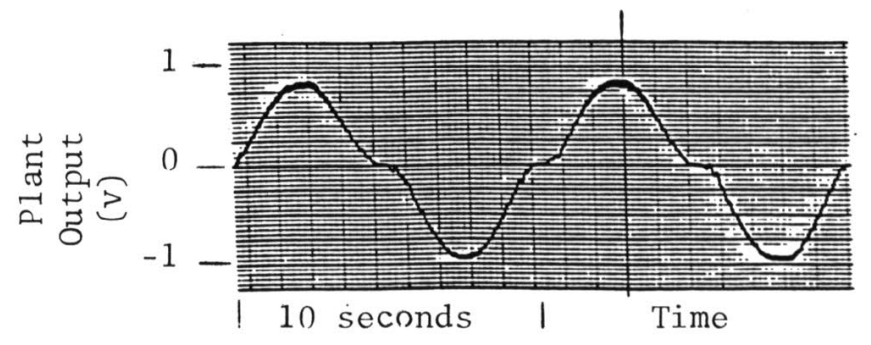

Initial Training

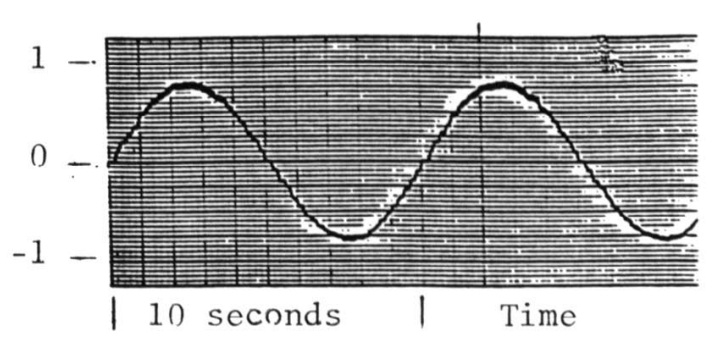

Final Training

(c) Least Squares
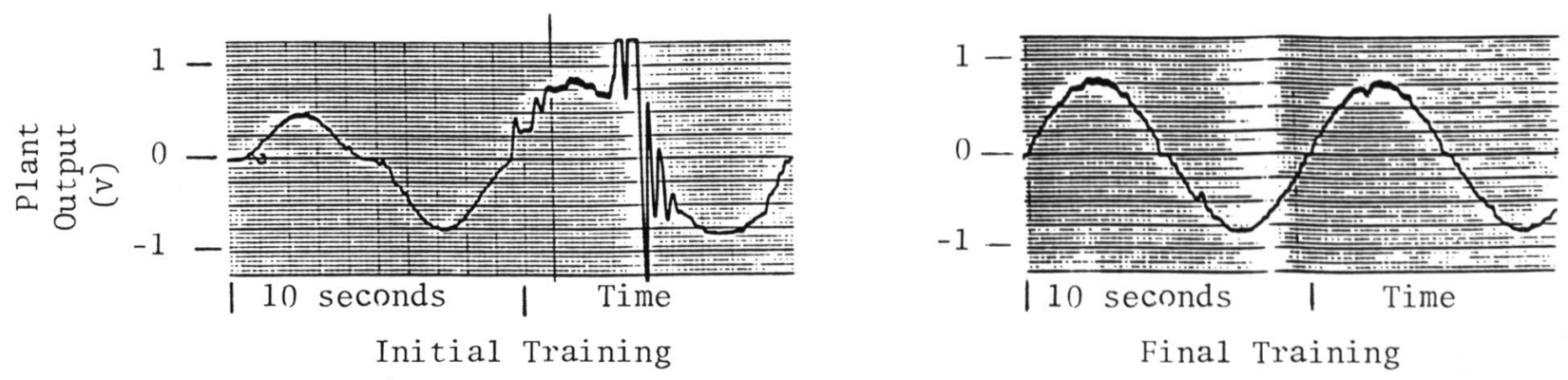

(d) Modified Newtons

Figure 5(c) and (d) Experimental Results (.1Hz)
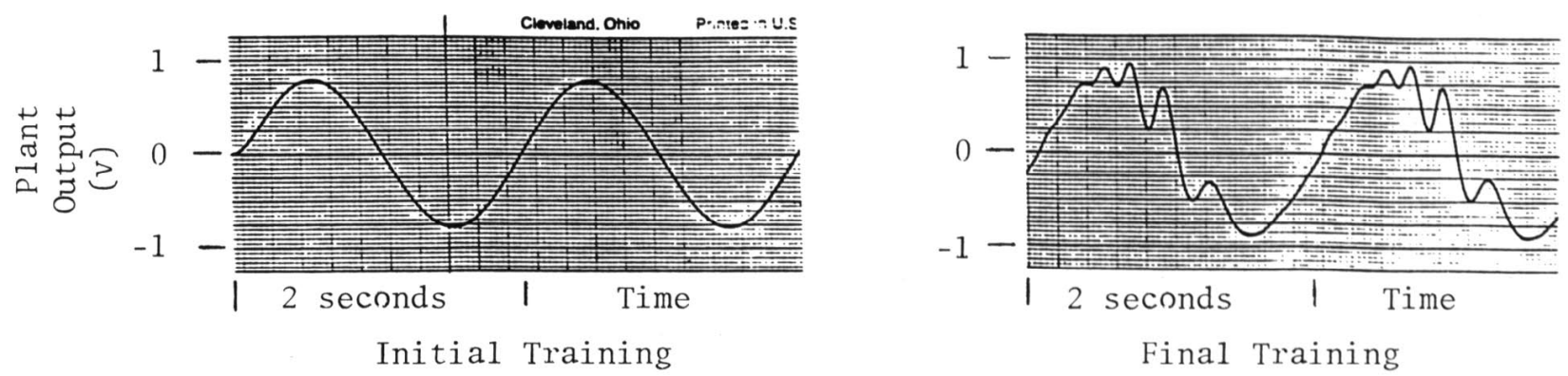

(a) Simulation

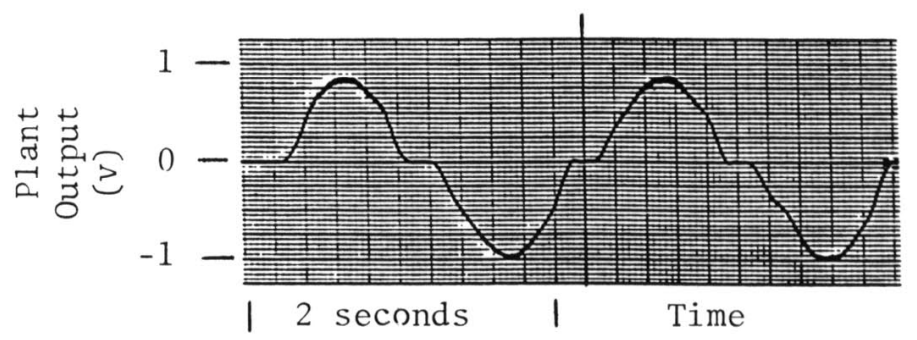

Initial Training

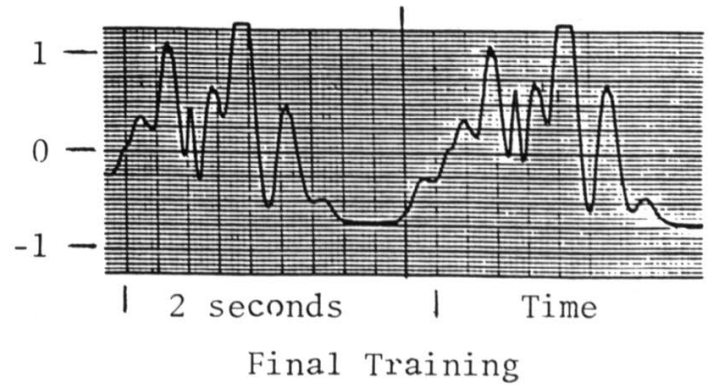

Final Training

(b) Experimental System

Figure 6(a) and (b) Simulated and Experimental Results $(0.5 \mathrm{~Hz}$, least squared approach) 